\title{
BAER-INVARIANTS OF ALGEBRAS
}

\author{
A. FRÖHLICH(1)
}

Explicitly this paper deals with functors of the category $\mathfrak{C}_{\Lambda}$ of associative algebras-not necessarily with identity-over a commutative ring $\Lambda$ with identity. The contents of the first part remain valid however in a much more general situation. We have formulated the theory of Baer-invariants in $\S \S 1-3$ in the language of $\Lambda$-algebras, in order to exhibit their significance in a concrete category and also in order to preserve the continuity with the later sections. The translations into abstract terms does not present any difficulty. It is in particular clear that the definitions and results of $\S \S 1-3$ apply equally well to any variety $\mathfrak{C}$ of groups with multiple operators in the sense of P. J. Higgins (cf. [3]).

With every subvariety $\mathfrak{B}$ of $\mathfrak{C}$ there is associated a subfunctor and a quotient functor of the identity functor of $\mathfrak{C}$ (cf. $\$ 1$, see also [5]). The absolute Baerinvariants appear then as their "derived" functors ( $\$ 3)$, the procedure involved being, however, quite different from that in [6]. The principal homological tool is that of a normal short complex (cf. \$2). Relative invariants are obtained by considering pairs of varieties $\mathfrak{B}_{1}$ and $\mathfrak{V}_{2}$ (cf. §3).

Historically one may take as starting point of the theory the discovery by Hopf (cf. [4]) that if

$$
G \cong F / K
$$

is a group, presented as a quotient group of a free group $F$, then the groups

$$
(F, F) /(F, K) \text { and }(F, F) \cap K /(F, K)
$$

are independent of the choice of $F$, i.e., are invariants of $G$. Hopf found in particular that

$$
(F, F) \cap K /(F, K)=H_{2}(G, Z)
$$

is the second homology group. In a series of papers (cf. [1]) Baer introduced a large class of invariants of type (2) derived from free, or reduced free presentations (1). It had long been felt that there is a deeper connection between Baer's work and the homological methods in algebra developed since.

Received by the editors June 23, 1962.

(1) The preparation of this paper was supported in part by the Office of Naval Research. 
In our terminology the original Baer-invariants are those associated with varieties $\mathfrak{C}$ of ordinary groups and "commutator" subvarieties $\mathfrak{P}$ of $\mathfrak{C}$. Baer's construction procedure is an iterative one, based on the commutator calculus. He was certainly aware that his methods could in principle be adapted, so as to yield invariants also in other situations. There is, however, a definite advantage in employing the homological procedure developed in this paper. It provides once and for all a universal, and at the same time technically quite transparent construction of Baer-invariants for any pair $\mathfrak{C}, \mathfrak{B}$, with $\mathfrak{C}$ say an arbitrary variety of groups with multiple operators and $\mathfrak{B}$ an arbitrary subvariety of $\mathfrak{C}$.

Elsewhere (cf. [6]) I have shown that the theory of derived functors and satellites in homological algebra can be extended to certain non-Abelian categories. The varieties of groups with multiple operators fall under this heading (cf. [6, II, Introduction]), and in fact some of the classical Baer-invariants were thus regained (cf. $[6, \mathrm{II}]$ ). The construction method there differs however fundamentally from the one used here. In [6] we deal with arbitrary additive functors and get derived functor or satellites in all dimensions. On the other hand for functors associated with varieties and for dimensions 0 and 1 the methods of the present paper go much deeper. The full connection between the two approaches remains yet to be explored.

From $\S 4$ onward we are specifically concerned with the category $\mathfrak{C}_{\Lambda}=\mathfrak{C}$ of $\Lambda$-algebras. In $\S 4$ we give a description of the Baer-invariants for varieties associated with ideals of $\Lambda$; these turn out to be tensor and torsion products.

Associating with each $\Lambda$-algebra $A$ the supplemented $\Lambda$-algebra $A^{+}$with augmentation ideal $A$, one obtains an isomorphism of $\mathfrak{C}$ with the category $\mathfrak{C}^{+}$of supplemented algebras. In $\S 5$ we study the Baer-invariants, and also the tensor product $A^{(n)}$ of $n$ copies of $A$ over $A^{+}$, from the point of view of supplemented algebras. In particular we show that if $A$ is a projective (free) $\Lambda$-algebra then, for all $n, A^{(n)}$ is a projective (free) $A^{+}$-module.

The subject matter of the last three sections are the powers of algebras, which play the same role here as the terms of the lower central series in group theory. The associated Baer-invariants are described in $\S 6$. It is shown that they can also be expressed by means of the functors $A^{(n)}$. In $\$ 7$ we establish a connection with the homology groups of supplemented algebras. As a particular case we obtain the exact algebra analogue to the Hopf equation (3) for $\mathrm{H}_{2}(G, Z)$. Finally, in $\S 8$ we consider some illustrative examples.

1. Varieties of algebras. Throughout $\Lambda$ is a fixed commutative ring with identity. A $\Lambda$-module is always one on which 1 acts as the identity map. A $\Lambda$-algebra $A$ is a ring and a $\Lambda$-module, the two additions to coincide, which satisfies the usual postulate 


$$
\lambda\left(a_{1} a_{2}\right)=\left(\lambda a_{1}\right) a_{2}=a_{1}\left(\lambda a_{2}\right), \quad \text { for all } a_{1}, a_{2} \in A \text {, all } \lambda \in \Lambda \text {. }
$$

The existence of an identity in $A$ is thus not to be assumed. The term "free algebra" is to be understood in this sense, as an algebra "without" identity. The category of $\Lambda$-algebras will be denoted by $\mathfrak{C}$.

We shall frequently speak simply of "modules" and of "algebras," the base ring being $\Lambda$, unless otherwise mentioned. If $A$ and $B$ are algebras, a "homomorphism" $f: A \rightarrow B$ is always an algebra-homomorphism, unless otherwise specified. The modules $\operatorname{Im} f$ and $\operatorname{Ker} f$ have again the structure of algebras. Moreover $\operatorname{Ker} f$ is an ideal of $A$, where by ideal we mean "ring ideal" plus " $\Lambda$-submodule."IIf also $\operatorname{Im} f$ is an ideal of $B$ we say that $f$ is normal. It is now clear what is meant by a monomorphism, an epimorphism, and an exact sequence in $\mathfrak{C}$.

We shall make use of the non-Abelian form of the basic lemma on connecting homomorphism (cf. [6, I, 4.1, 4.2]). Let

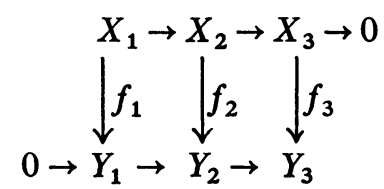

be a commutative diagram in $\mathfrak{C}$ with exact rows. The construction of a connecting homomorphism and the results given in [6, cf. I, \$4] for a non-Abelian category remain valid here. If in particular the $f_{i}$ are all normal then the $\Lambda$-modules Coker $f_{i}$ have the structure of algebras, and we have

\subsection{LemMa. The sequence in $\mathbb{C}$}

$$
\operatorname{Ker} f_{1} \rightarrow \operatorname{Ker} f_{2} \rightarrow \operatorname{Ker} f_{3} \rightarrow \operatorname{Coker} f_{1} \rightarrow \operatorname{Coker} f_{2} \rightarrow \operatorname{Coker} f_{3}
$$

is exact. If $X_{1} \rightarrow X_{2}$ is a monomorphism then so is $\operatorname{Ker} f_{1} \rightarrow \operatorname{Ker} f_{2}$. If $Y_{2} \rightarrow Y_{3}$ is an epimorphism then so is Coker $f_{2} \rightarrow \operatorname{Coker} f_{3}$.

We shall consider covariant functors $T$ of $\mathfrak{C}$ in $\mathfrak{C} . T$ associates with each algebra $A$ an algebra $T(A)$, and with each homomorphism $f: A \rightarrow B$ a homomorphism $T(f): T(A) \rightarrow T(B)$. In addition to the usual axioms

A. (i) $T(f g)=T(f) T(g)$,

(ii) $T\left(\iota_{A}\right)=\iota_{T(A)}$,

we also postulate

(iii) $T(0)=0$.

Here $\iota$, with various subscripts, stands for identity maps, and 0 is always a null map.

A variety $\mathfrak{B}$ in $\mathfrak{C}$ is a full subcategory of $\mathfrak{C}$, satisfying the following axioms:

B. (i) If $f: A \rightarrow B$ is an epimorphism and $A \in \mathfrak{B}$ then $B \in \mathfrak{B}$. 
(ii) If $f: A \rightarrow B$ is a monomorphism and $B \in \mathfrak{B}$, then $A \in \mathfrak{B}$.

(iii) If $\left\{A_{i}, f_{i}\right\}$ is an indexed set of pairs of algebras $A_{i} \in \mathfrak{B}$ and epimorphisms $f_{i}: A \rightarrow A_{i}$, with $A$ fixed in $\mathfrak{C}$, and if $\bigcap \operatorname{Ker} f_{i}=0$ then $A \in \mathfrak{B}$.

$\mathrm{B}$ (iii) can be replaced by

$\mathrm{B}(\mathrm{iiia})$ If $\left\{A_{i}\right\}$ is an indexed set of algebras $A_{i} \in \mathfrak{B}$ then $\prod A_{i} \in \mathfrak{B}$.

The two systems of axioms are equivalent here, and in fact in any category with direct products. B(i)-(iii) is, however, more general; it shows that the notion of a variety makes sense even without the existence of direct products.

For $A \in \mathbb{C}$ we write $V(A)=\bigcap \operatorname{Ker} \alpha, \alpha$ running through the homomorphisms $A \rightarrow B$ with $B \in \mathfrak{B} . V(A)$ is an ideal. Let $U(A)=A / V(A) . V$ and $U$ are functors, the subfunctor and the quotient functor associated with the given variety $\mathfrak{B}$. We then have (cf. $[5, \S 2])$

1.2. Theorem. (i) For all $A, U(A) \in \mathfrak{B}$. Also $A \in \mathfrak{B}$ if and only if $A=U(A)$, i.e., $V(A)=0$.

(ii) $A$ subfunctor $V$ of the identity functor of $\mathbb{C}$ is associated with a variety if and only if $V(A)$ is always an ideal of $A$, and $V$ preserves epimorphisms.

(iii) $A$ quotient functor $U$ of the identity functor of $\mathbb{C}$ is associated with a variety if and only if $U$ is right exact.

We denote by $A^{(n)}$ the $n$-fold direct product of copies of the algebra $A$ and by $g^{(n)}: A^{(n)} \rightarrow B^{(n)}$ the homomorphism induced by the homomorphism $g: A \rightarrow B$. A word $w$ in $\mathbb{C}$ (of length $n)(n \geqq 1)$ is a function which associates with every $A \in \mathbb{C}$ a map

$$
w_{A}: A^{(n)} \rightarrow A
$$

of sets, such that for every homomorphism $g: A \rightarrow B$ the diagram

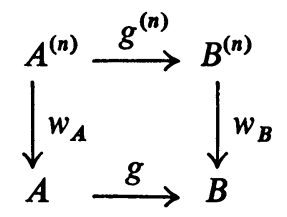

commutes. The elements $w_{A}(\bar{a})$ with $\bar{a} \in A^{(n)}$ will be called the values of $w_{A}$, or the values of $w$ in $A$. The word which, for all $A$, induces the zero map will be denoted by 0 , irrespective of its length.

We shall identify $A^{(r)} \times A^{(s)}=A^{(r+s)}$ and occasionally denote the values of a word $w$ of length $r+s$ by $w_{A}\left(\bar{a}, \bar{a}^{\prime}\right), \bar{a} \in A^{(r)}, \bar{a}^{\prime} \in A^{(s)}$, if the two sets of "variables" play distinct roles. If $w$ is a word of length $n$, we define words ${ }^{*} w$ and $w^{*}$ of length $n+1$ by

$$
\left.\begin{array}{c}
{ }^{*} w_{A}(a, \bar{a})=a w_{A}(\bar{a}) \\
w_{A}^{*}(\bar{a}, a)=w_{A}(\bar{a}) a
\end{array}\right\} \quad a \in A, \bar{a} \in A^{(n)} .
$$


Clearly $\left({ }^{*} w\right)^{*}=*\left(w^{*}\right)$. If $W$ is a set of words we write $\hat{W}$ for the set of all words $w, w^{*},{ }^{*} w,\left({ }^{*} w\right)^{*}$ with $w \in W$.

Let $W$ be a set of words. The algebras $A$ with $w_{A}=0_{A}$ for all $w \in W$ form a a variety $\mathfrak{B}_{W}$. Every variety $\mathfrak{B}$ is given in this manner. In fact let $W^{\mathfrak{B}}$ be the set of words $w$ with $w_{A}=0_{A}$ for all $A \in \mathfrak{B}$. Then for $W=W^{\mathfrak{B}}$ we have $\mathfrak{B}=\mathfrak{B}_{W}$. This choice of $W$ is, however, for practical purposes too big. Useful criteria here are given by:

1.3. LEMMA. Let $W$ be a set of words, and let $\mathfrak{B}$ be a variety with associated subfunctor $V$. Then $\mathfrak{B}=\mathfrak{V}_{W}$ if, and only if, for all $A \in \mathfrak{C}, V(A)$ is generated-as an ideal-by the values in $A$ of the words in $W$.

Suppose this to be the case. Then for all $A \in C, V(A)$ is generated-as a module already-by the values in $A$ of the words in $\hat{W}$.

Note here that if $W$ is finite then so is $\hat{W}$.

2. Short complexes. We consider complexes of algebras, whose differentiations are homomorphisms of algebras. A short complex (s. complex) $\mathbf{A}$ is one for which $A_{n}=0$, except when $n=0,1$. A normal short complex (n. s. complex) A is a short complex for which the map $A_{1} \rightarrow A_{0}$ is a normal monomorphism. As a rule we shall then view $A_{1}$ simply as an ideal of $A_{0}$. The homology complex of a n.s. complex $\mathbf{A}$ is then nontrivial at most in zero dimension, and we thus get associated with it the exact sequence

$$
0 \rightarrow A_{1} \stackrel{\alpha}{\rightarrow} A_{0} \stackrel{\varepsilon}{\rightarrow} H_{0}(\mathbf{A}) \rightarrow 0 .
$$

A word $w$ in the category of n.s. complexes, of length $(n, m)$, is a function which associates with every n.s. complex $\mathbf{A}$ a pair of maps of sets

$$
\begin{aligned}
& w_{\mathrm{A}}^{0}: A_{0}^{(n)} \rightarrow A_{0}, \\
& w_{\mathrm{A}}^{1}: A_{0}^{(n)} \times A_{1}^{(m)} \rightarrow A_{1}
\end{aligned}
$$

satisfying the obvious commutativity conditions analogous to (1.1). We associate with each word $v$ in the category of algebras, of length $n$, a word $v^{\prime}=w$ in the category of n.s. complexes, of length $(n, n)$ called the extension of $v . w$ is given by the rule

$$
\left.\begin{array}{ll}
w_{\mathrm{A}}^{0}\left(\bar{a}_{0}\right) & =v_{\Lambda_{0}}\left(\bar{a}_{0}\right), \\
w_{\mathrm{A}}^{\mathbf{1}}\left(\bar{a}_{0}, \bar{a}_{1}\right) & =v_{A_{0}}\left(\bar{a}_{0}+\bar{a}_{1}\right)-v_{A_{0}}\left(\bar{a}_{0}\right),
\end{array}\right\} \quad \bar{a}_{i} \in A_{i}^{(n)},
$$

where of course $A_{1}^{(n)}$ is considered as embedded in $A_{0}^{(n)}$. If first we view $w_{\mathbf{A}}^{1}$ as a map into $A_{0}$ we see that it is a difference word in the sense of Higgins (cf. [3]). Applying the epimorphism $\varepsilon$ of (2.1) to its values, we find that these do in fact lie in $\operatorname{Ker} \varepsilon=A_{1}$. It is now easily seen that $w$ is in fact a word. 
Let there be in the sequel $\mathfrak{B}$ a variety with associated functors $V$ and $U$, and let $W$ be a set of words whose values generate $V(A)$ as a module, for each $A \in \mathbb{C}$. Denote by $W^{\prime}$ the set of extensions $w=v^{\prime}$ of words $v \in W$. If $\mathbf{A}$ is an n.s. complex, write $V_{0}(\mathbf{A})$ for the ideal in $A_{0}$ generated by the values of the $w_{\mathbf{A}}^{0}$, and $V_{1}(\mathbf{A})$ for the ideal in $A_{0}$ generated by the values of the $w_{\mathrm{A}}^{1}, w$ running through $W^{\prime}$. Then

$$
\begin{aligned}
& V_{0}(\mathbf{A})=V\left(A_{0}\right), \\
& V\left(A_{1}\right) \subseteq V_{1}(\mathbf{A}) \subseteq V\left(A_{0}\right) \cap A_{1} .
\end{aligned}
$$

We obtain a n.s. complex $\mathrm{V}(\mathbf{A})$ with components $V_{1}(\mathbf{A})$ and $V_{0}(\mathbf{A}) . \mathrm{V}$ is easily seen to be a functor of n.s. complexes. We shall show presently that it does depend solely on the variety. The associated exact sequence is

$$
0 \rightarrow V_{1}(\mathbf{A}) \rightarrow V_{0}(\mathbf{A}) \stackrel{\varepsilon_{V}}{\rightarrow} H_{0}(\mathbf{V}(\mathbf{A})) \rightarrow 0 .
$$

2.1. THeOREM. Let $\mathbf{A}$ be a n.s. complex and let $\mathfrak{F}$ be the set of ideals $J$ of $A_{0}$ having the following properties:

(i) $J \subseteq V\left(A_{0}\right)$, i.e., there is an exact sequence

$$
0 \rightarrow J \rightarrow V\left(A_{0}\right) \stackrel{\varepsilon_{J}}{\rightarrow} C \rightarrow 0 .
$$

(ii) Whenever $B \in \mathbb{C}$ and $f, g: B \rightarrow A_{0}$ is a pair of homomorphisms such that $\varepsilon f=\varepsilon g$, then $\varepsilon_{J} V(f)=\varepsilon_{J} V(g)$.

Then

(a) $V_{1}(\mathbf{A}) \in \mathfrak{F}$.

(b) If $J \in \mathfrak{F}$ then $V_{1}(\mathbf{A}) \subseteq J$.

Property (a) will be fundamental throughout. (b) shows that $V_{1}(\mathbf{A})$ is the unique minimal ideal with property (a). Thus in fact $\mathbf{V}(\mathbf{A})$ has a characterisation which is quite independent of the choice of the set of words $W$.

Proof. (a) Let $v \in W$ (length $n$ ), $w=v^{\prime}$, and let $f, g: B \rightarrow A$ be homomorphisms with $\varepsilon f=\varepsilon g$. For $\bar{b} \in B^{(n)}$ we get $f^{(n)}(\bar{b})=g^{(n)}(\bar{b})+\bar{a}_{1}, \bar{a}_{1} \in A_{1}^{(n)}$ Hence $f\left(v_{B}(\bar{b})\right)=v_{A_{0}}\left(g^{(n)}(\bar{b})+\bar{a}_{1}\right)=g\left(v_{B}(\bar{b})\right)+w_{\mathrm{A}}^{1}\left(g^{(n)}(\bar{b}), \bar{a}_{1}\right)$. Thus

$$
\varepsilon_{V} V(g) b=\varepsilon_{V} V(f) b
$$

whenever $b$ is a value of $v_{B}$ for some $v \in W$. As these values generate $V(B)$ as a module, the equation will then hold for all $b \in V(B)$.

For (b) it will suffice to prove the following proposition:

Let $v \in W^{\mathfrak{B}}$ (length $n$ ), $v^{\prime}=w$. Let elements $\bar{a}_{0} \in A_{0}^{(n)}, \bar{a}_{1} \in A_{1}^{(n)}$ be given. Then there is an algebra $B$, an element $b \in V(B)$ and homomorphisms $f, g: B \rightarrow A_{0}$ with $\varepsilon f=\varepsilon g$ such that

$$
V(f) b=V(g) b+w_{\mathbf{A}}^{1}\left(\bar{a}_{0}, \bar{a}_{1}\right) .
$$


These requirements are in fact satisfied on taking $B$ as the free algebra on elements $b_{1}, \cdots, b_{n}, b=v_{B}\left(b_{1}, \cdots, b_{n}\right)$, and choosing $f$ and $g$ as the homomorphisms with

$$
f\left(b_{i}\right)=a_{i}+a_{i}^{i}, \quad g\left(b_{i}\right)=a_{i},
$$

where

$$
\bar{a}_{0}=\left(a_{1}, \cdots, a_{n}\right), \quad \bar{a}_{1}=\left(a_{1}^{\prime}, \cdots, a_{n}^{l}\right) .
$$

For each n.s. complex A we define a short complex $\mathbf{U}(\mathbf{A})$ by

$$
U_{0}(\mathbf{A})=U\left(A_{0}\right), \quad U_{1}(\mathbf{A})=A_{1} / V_{1}(\mathbf{A}) .
$$

Then $U_{1}(\mathbf{A}) \rightarrow U_{0}(\mathbf{A})$ is normal, and by 1.2 ,

$$
H_{0}(\mathrm{U}(\mathbf{A})) \cong U\left(H_{0}(\mathbf{A})\right) \text {. }
$$

For the homology in dimension 1 we get

$$
H_{1}(\mathbf{U}(\mathbf{A}))=V_{0}(\mathbf{A}) \cap A_{1} / V_{1}(\mathbf{A}) .
$$

We have an exact sequence

$$
0 \rightarrow \mathbf{V}(\mathbf{A}) \rightarrow \mathbf{A} \rightarrow \boldsymbol{U}(\mathbf{A}) \rightarrow 0,
$$

and so by 1.1 and (2.5) an exact sequence

$$
0 \rightarrow H_{1}(\mathbf{U}(\mathbf{A})) \rightarrow H_{0}(\mathbf{V}(\mathbf{A})) \rightarrow H_{0}(\mathbf{A}) \rightarrow U\left(H_{0}(\mathbf{A})\right) \rightarrow 0 .
$$

As $\operatorname{Ker}\left[H_{0}(\mathbf{A}) \rightarrow U\left(H_{0}(\mathbf{A})\right)\right]=V\left(H_{0}(\mathbf{A})\right)$, the significant part of (2.8) can be rewritten as an exact sequence

$$
0 \rightarrow H_{1}(\mathrm{U}(\mathbf{A})) \rightarrow H_{0}(\mathrm{~V}(\mathbf{A})) \rightarrow V\left(H_{0}(\mathbf{A})\right) \rightarrow 0 .
$$

The sequences (2.7)-(2.9) are functors of n.s. complexes. Moreover by 2.1 we have

2.2. Lemma. Two homomorphisms $F, G: \mathbf{A} \rightarrow \mathbf{B}$ of n.s. complexes induce the same homomorphism on sequences (2.8), (2.9) provided that $H_{0}(F)=H_{0}(G)$.

In conclusion of this section we derive twoimportant properties of n.s. complexes.

2.3. Proposition. If the sequence

$$
0 \rightarrow A_{1} \rightarrow A_{0} \stackrel{\mathcal{E}}{\rightarrow} H_{0}(\mathrm{~A}) \rightarrow 0
$$

splits then

$$
H_{1}(\mathrm{U}(\mathbf{A}))=0, \quad H_{0}(\mathbf{V}(\mathbf{A})) \cong V\left(H_{0}(\mathbf{A})\right) .
$$

Proof. Let $\mathrm{A}^{*}$ be the n.s. complex with $A_{0}^{*}=H_{0}(\mathrm{~A}), A_{1}^{*}=0$. We obtain homomorphisms $F: \mathbf{A} \rightarrow \mathbf{A}^{*}, G: \mathbf{A}^{*} \rightarrow \mathbf{A}$, where $F_{0}=\varepsilon$ and where $G_{0}$ is a homomorphism which splits the sequence in 2.3. $H_{0}(F G)$ and $H_{0}(G F)$ are identity maps, 
whence by $2.2, H_{1}(\mathrm{U}(\mathbf{A}))=H_{1}\left(\mathbf{U}\left(\mathbf{A}^{*}\right)\right)$. The latter algebra is null. The second isomorphism now follows from the exactness of (2.9).

2.4. THEOREM. For every n.s. complex A

$$
V_{0}(\mathrm{~A}) A_{1}+A_{1} V_{0}(\mathrm{~A}) \subseteq V_{1}(\mathrm{~A}) .
$$

Proof. Let $v \in W^{\mathfrak{B}}$ (length $n$ ), and let $w$ be the extension of ${ }^{*} v$. Denote by $\overline{0}$ the zero of $A_{1}^{(n)}$. If $\tilde{a} \in A_{0}^{(n)}, a \in A_{1}$ then

$$
a v_{A_{0}}(\bar{a})={ }^{*} v_{A_{0}}(0+a, \bar{a}+\overline{0})-{ }^{*} v_{A_{0}}(0, \bar{a})=w_{\mathrm{A}}^{1}((0, \bar{a}),(a, \overline{0})) \in V_{1}(\mathrm{~A}) \text {. }
$$

Thus we see that $A_{1} V_{0}(\mathrm{~A}) \subseteq V_{1}(\mathrm{~A})$, and similarly we get $V_{0}(\mathrm{~A}) A_{1} \subseteq V_{1}(\mathrm{~A})$.

An ideal $J$ of an algebra $A$ will be called a central ideal if $J$ is annihilated by $A$, i.e., if $A J+J A=0$. $A$ is a central ideal of $A$ precisely, when it has zero multiplication. The variety of $\mathfrak{C}$ of such algebras may be identified with the category of $\Lambda$-modules. A homomorphism $f: B \rightarrow A$, with $\operatorname{Im} f$ central in $A$, will be called central, and an exact sequence

$$
0 \rightarrow B \stackrel{f}{\rightarrow} A \rightarrow C \rightarrow 0
$$

will be called central, if $f$ is central. We then have

2.5. Corollary. (2.9) is a central sequence. In particular $H_{1}(\mathrm{U}(\mathbf{A})$ ) has zero multiplication.

3. The Baer-invariants. An isomorphism

$$
H_{0}(A) \cong A
$$

of algebras, where $\mathbf{A}$ is an n.s. complex with $A_{0}$ a projective algebra, is called a projective presentation of $A$. $A$ will always have a projective presentation. Moreover by 2.2 the homology algebras

$$
\begin{aligned}
& H_{0}(\mathrm{~V}(\mathrm{~A}))=D_{0} V(A), \\
& H_{1}(\mathrm{U}(\mathrm{A}))=D_{1} U(A)
\end{aligned}
$$

are already determined by $A$ to within isomorphism.

Let $f: A \rightarrow B$ be a homomorphism. Choose a projective presentation $H_{0}(\mathbf{B}) \cong B$ of $B$. Then there is a homomorphism $F: \mathbf{A} \rightarrow \mathbf{B}$ such that the diagram

$$
\begin{aligned}
& H_{0}(\mathrm{~A}) \cong A \\
& \downarrow H_{0}(F) \quad \downarrow \\
& H_{0}(\mathrm{~B}) \cong B
\end{aligned}
$$

commutes. By 2.2 the homomorphisms

$$
\begin{aligned}
& H_{0}(\mathrm{~V}(F))=D_{0} V(f), \\
& H_{1}(\mathrm{U}(F))=D_{1} U(f)
\end{aligned}
$$


will not depend on the particular choice of $F$. We now recognize $D_{0} V$ and $D_{1} U$ as functors in the sense of Definition A (\$1), the Baer-invariants of $\mathfrak{B}$. By 2.3 we have

3.1. Proposition. If $A$ is projective then

$$
D_{1} U(A)=0, \quad D_{0} V(A)=V(A) .
$$

From (2.8), (2.9) we now obtain exact sequences

$$
\begin{aligned}
& 0 \rightarrow D_{1} U(A) \rightarrow D_{0} V(A) \rightarrow A \rightarrow U(A) \rightarrow 0, \\
& 0 \rightarrow D_{1} U(A) \rightarrow D_{0} V(A) \rightarrow V(A) \rightarrow 0
\end{aligned}
$$

and by 2.5 the second sequence is central.

We consider now exact sequences

$$
0 \rightarrow C \stackrel{g}{\rightarrow} B \stackrel{f}{\rightarrow} A \rightarrow 0,
$$

and the associated commutative diagrams

$$
\begin{aligned}
& 0 \rightarrow \operatorname{Ker} D_{0} V(f) \rightarrow D_{0} V(B) \stackrel{D_{0} V(f)}{\longrightarrow} D_{0} V(A)
\end{aligned}
$$

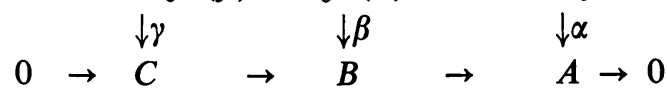

with exact rows. Here $\alpha$ and $\beta$ are homomorphisms occurring in sequence (3.4), and so are normal. Hence also $\gamma$ is normal.

The sequence (3.5) is said to be $\mathfrak{B}$-central if on setting $C=C_{1}, B=C_{0}$ we get for the n.s. complex $C$ the equation $V_{1}(C)=0$.

3.2. THEOREM. For every sequence (3.5), $D_{0} V(f)$ is an epimorphism, and so there exists a connecting homomorphism

$$
D_{1} U(A) \rightarrow \text { Coker } \gamma .
$$

If (3.5) is $\mathfrak{B}$-central then $\gamma$ is null, and so the sequence is exact.

$$
0 \rightarrow \operatorname{Ker} D_{0} V(f) \rightarrow D_{1} U(B) \rightarrow D_{1} U(A) \rightarrow C \rightarrow U(B) \rightarrow U(A) \rightarrow 0
$$

Proof. For some given projective presentation $H_{0}(\mathrm{~B}) \cong B$ of $B$, set $A_{0}=B_{0}$ and let $A_{1}$ be the kernel of the composition $A_{0} \rightarrow B \rightarrow A$. Then $H_{0}(\mathrm{~A}) \cong A$ is a projective presentation of $A$, and $D_{0} V(f)$ is the homomorphism

$$
V\left(B_{0}\right) / V_{1}(\mathbf{B}) \rightarrow V\left(A_{0}\right) / V_{1}(\mathbf{A}),
$$

induced by the identity map of $V\left(B_{0}\right)=V\left(A_{0}\right)$. It is therefore an epimorphism. Moreover its kernel is the quotient $V_{1}(\mathbf{A}) / V_{1}(\mathbf{B})$ of ideals of $B_{0}$. Hence if (3.5) is $\mathfrak{B}$-central, then $\gamma$ is null. The remainder of the theorem now follows from 1.1. 
One obtains relative Baer-invariants by considering side by side with $\mathfrak{B}$ a subvariety $\mathfrak{B}^{\prime}$ of $\mathfrak{B}$. Denoting by $I$ the identity functors of $\mathfrak{C}$ we obtain a commutative diagram with exact and normal rows and columns

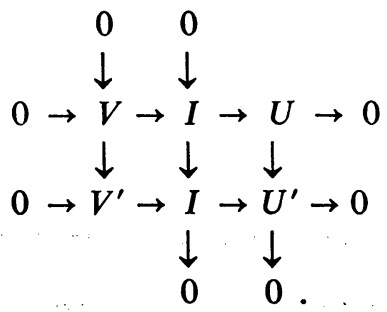

Going over to n.s. complexes, we derive a functor monomorphism $\mathbf{V} \rightarrow \mathbf{V}^{\prime}$ and a functor epimorphism $\mathbf{U} \rightarrow \mathbf{U}^{\prime}$, and so a commutative diagram with exact and normal rows and columns

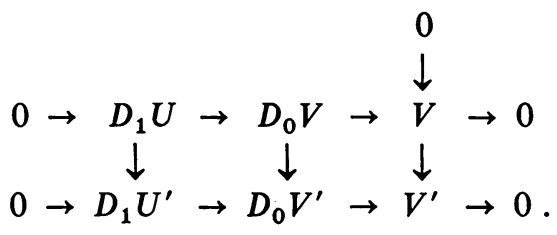

From (3.7) we obtain an isomorphism

$$
\operatorname{Ker}\left[U \rightarrow U^{\prime}\right] \cong \operatorname{Coker}\left[V \rightarrow V^{\prime}\right],
$$

and from (3.8) an isomorphism

$$
\operatorname{Ker}\left[D_{1} U \rightarrow D_{1} U^{\prime}\right] \cong \operatorname{Ker}\left[D_{0} V \rightarrow D_{0} V^{\prime}\right],
$$

together with an exact sequence

$$
0 \rightarrow \operatorname{Coker}\left[D_{1} U \rightarrow D_{1} U^{\prime}\right] \rightarrow \operatorname{Coker}\left[D_{0} V \rightarrow D_{0} V^{\prime}\right] \rightarrow \operatorname{Coker}\left[V \rightarrow V^{\prime}\right] \rightarrow 0 .
$$

4. Varieties induced by ideals of $\Lambda$. Let $J$ be an ideal of $\Lambda$. The algebras which qua modules are annihilated by $J$ form a variety $\mathfrak{B}$. The associated subfunctor is given by

$$
V(A)=J A \text {. }
$$

If $A, B$ are algebras then $A \otimes_{\Lambda} B$ has the structure of an algebra, irrespective of the existence of identities in $A$ or $B$. For the quotient functor $U$ associated with $\mathfrak{B}$ we get the natural isomorphism

$$
U(A) \cong \Lambda / J \otimes_{\Lambda} A \text {. }
$$

The isomorphism (4.2) for modules was established in [6, cf. II (6.5)]; it is easy to verify that it is in fact an isomorphism of algebras. For the Baer-invariants we get 
4.1. Proposition. There are natural isomorphisms

(i) $D_{0} V(A) \cong J \otimes_{\Lambda} A$,

(ii) $D_{1} U(A) \cong \operatorname{TOR}_{1}^{\Lambda}(\Lambda / J, A)$

of algebras.

Proof. Each $\lambda \in \Lambda$ defines a word $\bar{\lambda}$ given by the values $\bar{\lambda}_{A}(a)=\lambda a . V(A)$ is always generated as a module by the values of the $\bar{\lambda}$ in $A$, (all $\lambda \in J$ ). But the $\bar{\lambda}_{A}$ are homomorphisms of Abelian groups (in fact of $\Lambda$-modules). It follows easily now by considering the extensions of these words that for every n.s. complex A

$$
V_{1}(\mathbf{A})=V\left(A_{1}\right)
$$

Let $H_{0}(\mathbf{A}) \cong A$ be a projective presentation. The preceding argument shows that

$$
D_{0} V(A)=\operatorname{Coker}\left[J A_{1} \rightarrow J A_{0}\right] \text {. }
$$

We thus obtain a commutative diagram

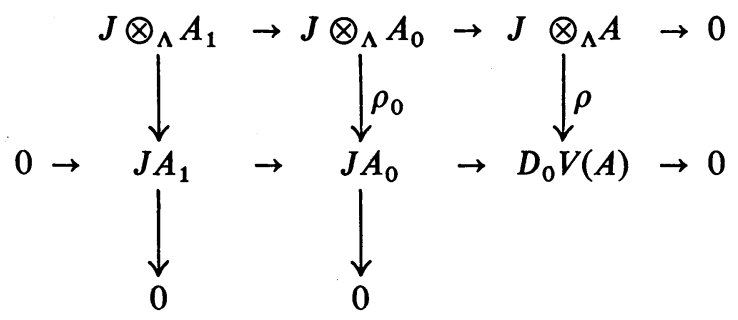

with exact rows and columns. $A_{0}$ is projective as a $\Lambda$-module and so $\operatorname{Ker} \rho_{0}=0$. Hence $\rho$ is an isomorphism. This establishes (i).

We have the identification $\operatorname{Ker}\left[J \otimes_{\Lambda} A \rightarrow A\right]=\operatorname{TOR}_{1}^{\Lambda}(\Lambda / J, A)$ of modu es. Taking the right hand side as algebra with zero multiplication, this becomes an identification of algebras. But now (ii) will follow from (i).

5. Algebras and supplemented algebras. We consider side by side with $\mathfrak{C}$ the category $\mathfrak{C}^{+}$of supplemented algebras (over $\Lambda$ ) (cf. [2, Chapter X]). For $A \in \mathbb{C}$ we make the direct sum of modules

$$
A^{+}=A+\Lambda
$$

into an algebra, the product being defined by

$$
\left(a_{1}, \lambda_{1}\right)\left(a_{2}, \lambda_{2}\right)=\left(a_{1} a_{2}+\lambda_{1} a_{2}+\lambda_{2} a_{1}, \lambda_{1} \lambda_{2}\right) .
$$

he map $A^{+} \rightarrow \Lambda$, given by the direct product (5.1), makes $A^{+}$into a supplemented algebra with augmentation ideal $A$. Conversely, if $A^{\prime}$ is a supplemented 
algebra with augmentation ideal $A$ then there is an isomorphism $A^{+} \cong A^{\prime}$ of supplemented algebras, inducing the identity map on $A$ and $\Lambda$. The two categories $\mathfrak{C}$ and $\mathfrak{C}^{+}$are now easily seen to be isomorphic, and any functor of one may be viewed as a functor of the other.

By a two sided $A^{+}$-module $M$ we mean a left and right $A^{+}$-module, the identity acting as the identity map, such that

$$
\left(a_{1} u\right) a_{2}=a_{1}\left(u a_{2}\right), \quad \text { for all } a_{1}, a_{2} \in A^{+} \text {, all } u \in M \text {. }
$$

The structure of a two sided $A^{+}$-module on a $\Lambda$-module $M$ is given by homomorphisms

$$
M \otimes_{\Lambda} A^{+} \rightarrow M, \quad A^{+} \otimes_{\Lambda} M \rightarrow M
$$

of $\Lambda$-modules, satisfying the obvious identities.

A functor $T$ of $\mathbb{C}$ whose values are $\Lambda$-modules is said to admit a two sided module structure (over $\mathbb{C}^{+}$) if:

C. (i) for each $A \in \mathbb{C}, T(A)$ has the structure of a two sided $A^{+}$-module. This structure is given by homomorphisms

$$
T(A) \otimes_{\Lambda} A^{+} \rightarrow T(A), \quad A^{+} \otimes_{\Lambda} T(A) \rightarrow T(A) .
$$

We now require in addition:

(ii) If $f: A \rightarrow B$ is a homomorphism of algebras and $f^{+}: A^{+} \rightarrow B^{+}$the induced homomorphism of supplemented algebras then the diagrams

(a)

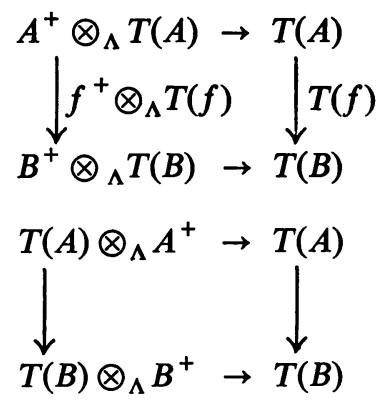

commute.

An algebra $A$, the supplemented algebra $A^{+}$, all ideals of $A$, and their quotient algebras are two sided $A^{+}$-modules. Hence the functors $A^{+}$, the identity functor $I$ of $\mathfrak{C}$, and the functors $U, V$, associated with a variety of $\mathfrak{C}$, admit a two sided module structure. The same is true for the functor (3.9) associated with a pair of varieties. Moreover we have in the notation of $\$ 3$ : 
5.1. Proposition. The Baer-invariants $D_{0} V, D_{1} U$ and the relative Baerinvariants $\operatorname{Ker}\left[D_{1} U \rightarrow D_{1} U^{\prime}\right]$, Coker $\left[D_{1} U \rightarrow D_{1} U^{\prime}\right]$, Coker $\left[D_{0} V \rightarrow D_{0} V^{\prime}\right]$ admit a two sided module structure over $\mathfrak{C}^{+}$.

For fixed A the maps of (3.4) and (3.7)-(3.11) are homormophisms of two sided $A^{+}$-modules.

Proof. Choose a projective presentation $H_{0}(\mathrm{~A}) \cong A$. Then

$$
D_{1} U(A)=H_{1}(\mathrm{U}(\mathbf{A})), \quad D_{0} V(A)=H_{0}(\mathrm{~V}(\mathbf{A}))
$$

have the structure of two sided $A_{0}^{+}$-modules and the map $D_{1} U(A) \rightarrow D_{0} V(A)$ preserves this structure. But by $2.4, A_{1}$ annihilates both $D_{0} V(A)$ and $D_{1} U(A)$ on the left and on the right. Hence these two sided $A_{0}^{+}$-modules have in fact the structure of two sided $A^{+}$-modules, and this structure is independent of the choice of the particular projective presentation.

Similar arguments give the whole of Proposition 5.1.

If $M$ and $N$ are two sided $A^{+}$-modules then so are $M \otimes_{\Lambda} N$ and $M \otimes_{A^{+}} N$, the left (right) $A^{+}$-module structure to be taken from $M$ (and $N$ ). Moreover, the map

$$
M \otimes_{\Lambda} N \rightarrow M \otimes_{A^{+}} N
$$

is a natural epimorphism.

5.2. Lemma. Let $T_{1}, T_{2}$ be functors of $\mathbb{C}$ admitting a two sided module structure. Write

$$
\begin{aligned}
T_{1} \otimes_{\Lambda} T_{2}(A) & =T_{1}(A) \otimes_{\Lambda} T_{2}(A), \\
T(A) & =T_{1}(A) \otimes_{A^{+}} T_{2}(A) .
\end{aligned}
$$

Then $T_{1} \otimes_{\Lambda} T_{2}$ and $T$ are functors of $\mathbb{C}$ admitting a two sided module structure.

Proof. The assertion on $T_{1} \otimes_{\Lambda} T_{2}$ is trivial. It thus remains to be shown that given a homomorphism $f: A \rightarrow B$ of algebra there is one and only one homomorphism

$$
T(f): T(A) \rightarrow T(B)
$$

of $\Lambda$-modules such that the diagrams (a) and (b) in $C$, (ii) and the diagram

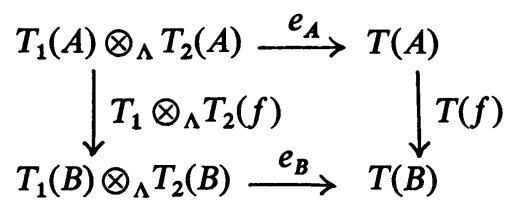

commute. 
We first consider diagram (c). The $\Lambda$-module $\operatorname{Ker} e_{A}$ is generated by the elements

$$
x_{1} a \otimes x_{2}-x_{1} \otimes a x_{2}, \quad x_{1} \in T_{1}(A), x_{2} \in T_{2}(A), a \in A^{+} .
$$

$T_{1} \otimes_{\Lambda} T_{2}(f)$ maps these generators, and therefore the whole module $\operatorname{Ker} e_{A}$ into $\operatorname{Ker} e_{B}$. As $e_{A}$ is an epimorphism it follows that there is one and only one homomorphism $T(f)$ making (c) commutative. The commutativity of (a) and (b) is now a consequence of the commutativity of the corresponding diagrams, with $T$ replaced by $T_{1} \otimes_{\Lambda} T_{2}$.

Write from now on

$$
A^{(0)}=A^{+}, \quad A^{(1)}=A, \quad A^{(n+1)}=A^{(n)} \otimes_{A^{+}} A .
$$

The symbol $A^{(n)}$ has thus henceforth quite a different connotation from that in $\S 2$; the direct products to which it had referred then will no longer occur. We shall always identify

$$
A^{(r+s)}=A^{(r)} \otimes_{A^{+}} A^{(s)} \quad \text { for } r \geqq 0, s \geqq 0 .
$$

By 5.2 we obtain, for each $r$, a functor $I^{(r)}$ of $\mathfrak{C}$ admitting a two sided module structure over $\mathfrak{C}^{+}$. Its values are $I^{(r)}(A)=A^{(r)}$.

Let $n \geqq m \geqq 1$, and $1<i_{1}<i_{2}<\cdots<i_{m-1}<n$.

We define a homomorphism

$$
g_{m . A}^{n}: A^{(n)} \rightarrow A^{(m)}
$$

by

$$
g_{m, A}^{n}\left(a_{1} \otimes a_{2} \otimes \cdots \otimes a_{n}\right)=a_{1} \cdots a_{i_{1}-1} \otimes a_{i_{1}} \cdots a_{i_{2}-1} \otimes \cdots \otimes a_{i_{m-1}} \cdots a_{n} .
$$

Here the right hand side, and so the definition of $g_{m, A}^{n}$ is in fact independent of the choice of $i_{1}, \cdots, i_{m-1}$. Next let

$$
g_{0, A}^{0}: A^{+} \rightarrow A^{+}
$$

be the identity map, and

$$
g_{0, A}^{1}: A=A^{(1)} \rightarrow A^{(0)}=A^{+}
$$

the inclusion map. Finally if $n>0$ set

$$
g_{0, A}^{n}=g_{0, A}^{1} g_{1, A}^{n} \text {. }
$$

Then for $n \geqq r \geqq 0, g_{r, A}^{n}$ is a homomorphism of two sided $A^{+}$-modules, giving rise to a homomorphism

$$
g_{r}^{n}: I^{(n)} \rightarrow I^{(r)}
$$

of functors. 
Denote by $\iota_{A}^{n}$ the identity map of $A^{(n)}$. Then

$$
\iota^{n}=g_{n}^{n}
$$

and

$$
i_{A}^{s} \otimes_{A^{+}} g_{1, A}^{n-r+1} \otimes_{A^{+}} l_{A}^{t}=g_{r, A}^{n} \quad(s \geqq 0, t \geqq 0, s+t=r-1) .
$$

From the definition we now have

$$
g_{r}^{n} g_{n}^{m}=g_{r}^{m} \quad(0 \leqq r \leqq n \leqq m) .
$$

Also

$$
g_{l, A}^{n} \otimes_{A^{+}} g_{q, A}^{m}=\left(\iota_{A}^{l} \otimes_{A^{+}} g_{q, A}^{m}\right)\left(g_{l, A}^{n} \otimes_{A^{+}} \iota_{A}^{m}\right)
$$

and so from (5.2)-(5.4)

$$
g_{l, A}^{n} \otimes_{A^{+}} g_{q, A}^{m}=g_{l+q, A}^{n+m} \quad(l \leqq n, q \leqq m) .
$$

Denote by $e_{A}^{n}$ the homomorphism

The map

$$
A^{(n)} \otimes_{\Lambda} A^{(n)} \rightarrow A^{(n)} \otimes_{A^{+}} A^{(n)}
$$

$$
h_{A}^{n}=g_{n, A}^{2 n} e_{A}^{n}: A^{(n)} \otimes_{\Lambda} A^{(n)} \rightarrow A^{(n)}
$$

now defines on $A^{(n)}$ the structure of an algebra, which for $n=0,1$ coincides with the given one of $A^{(0)}=A^{+}$and $A^{(1)}=A$.

Omitting in the following calculation the subscript $A$ we now get for $m \geqq n \geqq 1$, by (5.4) and (5.5),

$$
\begin{aligned}
h^{n}\left(g_{n}^{m} \otimes_{\Lambda} g_{n}^{m}\right) & =g_{n}^{2 n} e^{n}\left(g_{n}^{m} \otimes_{\Lambda} g_{n}^{m}\right)=g_{n}^{2 n}\left(g_{n}^{m} \otimes_{A^{+}} g_{n}^{m}\right) e^{m} \\
& =g_{n}^{2 n} g_{2 n}^{2 m} e^{m}=g_{n}^{m} \dot{g}_{m}^{2 m} e^{m}=g_{n}^{m} h^{m} .
\end{aligned}
$$

Thus $g_{n, A}^{m}$ is a homomorphism of algebras. The same is also true for $n=0$; one only has to look at the case $m=1, n=0$.

We have already seen that $g_{m, A}^{2 m}$ defines a functor homomorphism $I^{(2 m)} \rightarrow I^{(m)}$. We can reinterpret this statement to say that, whenever $f: A \rightarrow B$ is a homomorphism of algebras, then so is $I^{(m)}(f)$.

We recapitulate:

5.3. Proposition. The modules $A^{(n)}$ have the structure of algebras and define a functor $I^{(n)}$ of $\mathfrak{C}$ in $\mathfrak{C}$ which admits a two sided module structure over $\mathfrak{C}^{+}$.

The maps $g_{n, A}^{m}(m \geqq n)$ are homomorphisms of algebras and of two sided $A^{+}$-modules, defining a homomorphism $g_{n}^{m}: I^{(m)} \rightarrow I^{(n)}$ of functors.

Now we prove

5.4. THEOREM. If $A$ is a projective algebra then, for all $n, A^{(n)}$ is a projective right $A^{+}$-module and a projective left $A^{+}$-module, and for all $m \geqq n$, $g_{n, A}^{m}$ is a monomorphism. 
If, moreover, $A$ is a free algebra then, for all $n, A^{(n)}$ is a free right $A^{+}$-module, and a free left $A^{+}$-module.

Proof. Suppose that $A$ is a projective algebra. We shall first show that it is a projective right $A^{+}$-module.

Let $f: M \rightarrow A$ be an epimorphism of right $A^{+}$-modules. We have to establish the existence of a homomorphism $g: A \rightarrow M$ of right $A^{+}$-modules such that $f g=\iota_{A}$.

Define a multiplication in $M$ by

$$
m_{1} m_{2}=m_{1} f\left(m_{2}\right),
$$

where the element $f(m)$ of $A$ is viewed as an element of $A^{+}$. One verifies that $M$ has now the structure of a $\Lambda$-algebra and that $f$ becomes a homomorphism of algebras. Therefore there is a homomorphism $g: A \rightarrow M$ of algebras such that $f g=\iota_{A}$. But then $g$ must be also a homomorphism of right $A^{+}$-modules.

Similarly one shows that $A$ is a projective left $A^{+}$-module. The assertion on $A^{(n)}$ now follows easily by induction, using elementary properties of the tensor product.

$g_{0, A}^{1}$ is always a monomorphism. By (5.4) we only have to show that also $g_{n, A}^{n+1}$ is a monomorphism, for $n \geqq 0$. But $g_{n, A}^{n+1}=\iota_{A}^{n} \otimes_{A}+g_{0, A}^{1}$ is induced by the monomorphism $A \rightarrow A^{+}$. $A^{(n)}$ being a projective right $A^{+}$-module it follows that $g_{n A}^{n+1}$ is in fact a monomorphism.

Let $A^{n}$ be the ideal of $A$ generated by the products of $n$ factors $(n \geqq 1)$. Then $A^{n}=\operatorname{Im} g_{1, A}^{n}$. Now suppose that $A$ is a free algebra. Then, by the first part of $5.4, A^{(n)} \cong A^{n}$. We shall prove then that $A^{n}$ is a free left $A^{+}$-module. The same is of course trivially true for $A^{(0)}$.

Let $A$ be the free algebra with the indexed set $\left\{a_{j}\right\}(j \in J)$ as a free generating set. Let $J_{n}$ be the set of $n$-tuples $k=\left(j_{1}, \cdots, j_{n}\right)$ in $J$, and put, for $k \in J_{n}$,

$$
a_{k}=a_{j_{1}} a_{j_{2}} \cdots a_{i_{n}} \text {. }
$$

Let for all $n \geqq 1, A_{n}$ be the free $\Lambda$-module on the $a_{k}\left(k \in J_{n}\right)$, and let $A_{0}=\Lambda$. Then

$$
A=\sum_{n=1}^{\infty} A, \quad A^{+}=\sum_{n=0}^{\infty} A_{n}
$$

are graded algebras with the strong multiplicative property

$$
A_{n} A_{m}=A_{n+m} \text {. }
$$

If $n \geqq 1, m \geqq 1$ the set $\left\{a_{k}\right\}\left(k \in J_{n+m}\right)$ coincides with the set $\left\{a_{l} a_{r}\right\}\left(l \in J_{n}, r \in J_{m}\right)$ and so $A_{n+m}$ is the direct sum of $\Lambda$-modules $a_{l} A_{m}\left(l \in J_{n}\right)$. The same is also true for $m=0$. But, as $A^{n}=\sum_{m=0}^{\infty} A_{n+m}$, we now see that $A^{n}$ is the direct sum of $\Lambda$-modules $a_{l} \sum_{m=0}^{\infty} A_{m}=a_{l} A^{+}$. The latter modules are in fact right $A^{+}$-modules, and as $A$ is a free algebra 


$$
a_{l} A^{+} \cong A^{+} \text {(isomorphism of right } A^{+} \text {-modules). }
$$

Hence finally $A^{n}$ is the direct sum of copies of $A^{+}$.

6. The powers of an algebra. For each positive integer $r$, let $\mathfrak{B}^{r}$ be the variety of nilpotent algebras of class $r-1$, i.e., of algebras in which all products of $r$ factors vanish. Thus $\mathfrak{B}^{(1)}$ consists of the zero algebra only, and $\mathfrak{B}^{(2)}$ is the category algebras with zero multiplication, i.e., of modules.

Denote by $V^{r}, U^{r}$ the associated functors. Let $A^{r}$ be the submodule of an algebra $A$ generated by the products

$$
a_{1} a_{2} \cdots a_{r}=w_{A}^{r}\left(a_{1}, a_{2}, \cdots, a_{r}\right) .
$$

Then

$$
V^{r}(A)=A^{r}, \quad U^{r}(A)=A / A^{r} .
$$

Let $\mathbf{A}$ be an n.s. complex. As $V^{r}(A)$ is always generated as a module by the values in $A$ of the word $w^{r}$, defined in (6.1), it follows that $V_{1}^{r}(\mathbf{A})$ is the module generated by products $a_{1} a_{2} \cdots a_{r}$ in $A_{0}$ with at least one factor out of $A_{1}$, i.e., we have

$$
\begin{aligned}
V_{1}^{1}(\mathbf{A}) & =A_{1}, \quad V_{1}^{2}(\mathbf{A})=A_{0} A_{1}+A_{1} A_{0}, \\
V_{1}^{r+1}(\mathbf{A}) & =A_{0}^{r} A_{1}+A_{0}^{r-1} A_{1} A_{0}+\cdots+A_{1} A_{0}^{r}
\end{aligned}
$$

(sum of submodules). We thus get from (6.2), (6.3) the recursion formulae

$$
\begin{aligned}
& V^{r+1}(A)=A V^{r}(A)=V^{r}(A) A, \\
& V_{1}^{r+1}(\mathbf{A})=A_{0}^{r} A_{1}+V_{1}^{r}(\mathbf{A}) A_{0}=A_{1} A_{0}^{r}+A_{0} V_{1}^{r}(\mathbf{A}) .
\end{aligned}
$$

Let now $H_{0}(\mathrm{~A}) \cong A$ be a projective presentation of $A$. Then

$$
\begin{aligned}
& D_{0} V^{r}(A)=A_{0}^{r} / V_{1}^{r}(\mathbf{A}), \\
& D_{1} U^{r}(A)=A_{0}^{r} \cap A_{1} / V_{1}^{r}(\mathbf{A}) .
\end{aligned}
$$

$\mathfrak{B}^{r}$ is a subvariety of $\mathfrak{B}^{r+1}$. We are thus in the situation discussed in $\S 3$, and we get the explicit formulae

$$
\begin{aligned}
\operatorname{Ker}\left[U^{r+1}(A) \rightarrow U^{r}(A)\right] & =A^{r} / A^{r+1}, \\
\operatorname{Ker}\left[D_{0} V^{r+1}(A) \rightarrow D_{0} V^{r}(A)\right] & =\left(A_{0}^{r+1} \cap V_{1}^{r}(\mathbf{A})\right) / V_{1}^{r+1}(\mathbf{A}), \\
\operatorname{Coker}\left[D_{0} V^{r+1}(A) \rightarrow D_{0} V^{r}(A)\right] & =A_{0}^{r} /\left(A_{0}^{r+1}+V_{1}^{r}(\mathbf{A})\right), \\
\operatorname{Coker}\left[D_{1} U^{r+1}(A) \rightarrow D_{1} U^{r}(A)\right] & =\left[A_{0}^{r+1}+\left(A_{0}^{r} \cap A_{1}\right)\right] /\left(A_{0}^{r+1}+V_{1}^{r}(\mathbf{A})\right) .
\end{aligned}
$$

Return now to the functor $I^{(r)}$ of $\S 5$. We have an exact sequence $0 \rightarrow \operatorname{Ker} g_{1}^{r} \rightarrow I^{(r)} \rightarrow I \rightarrow \operatorname{Coker} g_{1}^{r} \rightarrow 0$, whose maps, for fixed $A$, are homomorphisms of algebras and of two sided $A^{+}$-modules. 
6.1. THEOREM. There are unique functor isomorphisms

$$
\begin{aligned}
& t: \text { Coker } g_{1}^{r} \cong U^{r}, \\
& s: I^{(r)} \cong D_{0} V^{r}, \\
& u: \operatorname{Ker} g_{1}^{r} \cong D_{1} U^{r}
\end{aligned}
$$

such that the diagram

(D)

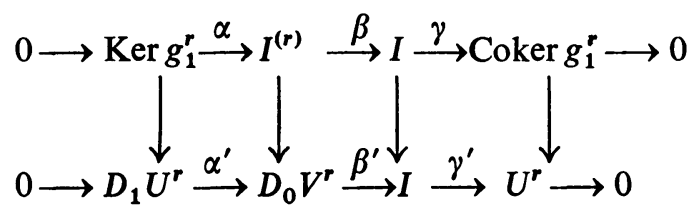

commutes. The maps $t_{A}, s_{A}, u_{A}$ are homomorphisms of algebras and of two sided $A^{+}$-modules.

Proof. Let $H_{0}(\mathrm{~A}) \cong A$ be a projective representation of $A$. By Theorem 5.4, $g_{1, A_{0}}^{r}$ gives rise to an isomorphism $A_{0}^{(r)} \cong A_{0}^{r}$ of algebras and of two sided $A^{+}$modules. We shall show later that this in turn induces an isomorphism

$$
\operatorname{Ker}\left[A_{0}^{(r)} \rightarrow A^{(r)}\right] \cong V_{1}^{r}(\mathbf{A}),
$$

on the respective ideals. Taking quotients we obtain an isomorphism

$$
s_{A}: A^{(r)} \cong D_{0} V^{(r)}(\mathbf{A})
$$

of algebras, and of two sided $A_{0}^{+}$-modules, i.e., of two sided $A^{+}$-modules. The functor property of the maps $S_{A}$ is then established in the usual manner.

The lower row of the diagram $D_{A_{0}}$, obtained from $D$ by "specialization" to $A_{0}$, is exact. By 3.1, $\beta_{A_{0}}^{\prime}$ is therefore a monomorphism and so the commutativity of $D_{A_{0}}$ determines $S_{A_{0}}$ uniquely. Moreover, the diagram

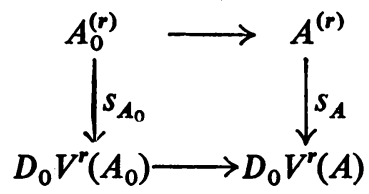

commutes. Its upper row being an epimorphism this determines $s_{A}$ uniquely.

As $\operatorname{Im} g_{1, A}^{r}=V^{r}(A)$ we have an isomorphism $t_{A}$ :Coker $g_{1, A}^{r} \cong U^{r}(A)$ of algebras and of two sided $A^{+}$-modules with the evident commutativity property. As $\gamma_{A}$ is an epimorphism this determines $t_{A}$ uniquely. Its functor property is immediate.

The existence and uniqueness of $u$, and all its properties now follow from the exactness of the rows of $D$. 
To establish (6.7) we proceed by induction, the case $r=1$ being trivial. For the step from $r$ to $r+1$ we consider the commutative diagram with exact rows and columns

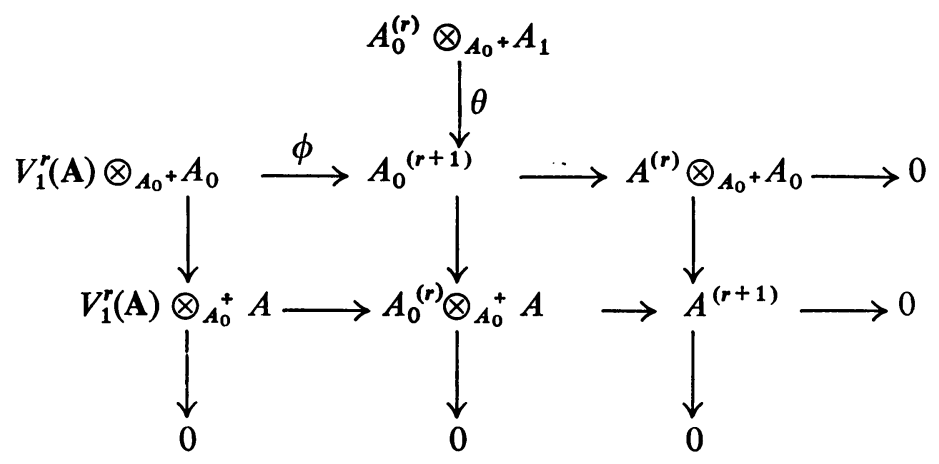

We get

$$
\operatorname{Ker}\left[A_{0}^{(r+1)} \rightarrow A^{(r+1)}\right]=\operatorname{Im} \phi+\operatorname{Im} \theta .
$$

But the latter ideal is mapped onto $V_{1}^{r}(\mathrm{~A}) A_{0}+A_{0}^{r} A_{1}$ under the isomorphism $A_{0}^{(r+1)} \cong A_{0}^{r+1}$. By (6.4) we now get (6.7) for $r+1$.

7. Powers and homology of supplemented algebras. The $n$th homology group of $A^{+}$with coefficients in the right $A^{+}$-module $M$ is $\operatorname{TOR}_{n}^{A^{+}}(M, \Lambda)$. We shall show that

$$
T(A)=\operatorname{TOR}_{n}^{A^{+}}\left(A^{(r)}, \Lambda\right)
$$

is a functor of $\mathfrak{C}$ with values in the category of $\Lambda$-modules. Let $f: A \rightarrow B$ be a homomorphism of algebras. Then $B^{(r)}$ has the structure of a right $A^{+}$-module and $f^{(r)}: A^{(r)} \rightarrow B^{(r)}$ is a homomorphism of right $A^{+}$-modules. We obtain a homomorphism

$$
\operatorname{TOR}_{n}^{A^{+}}\left(A^{(r)}, \Lambda\right) \rightarrow \operatorname{TOR}_{n}^{A^{+}}\left(B^{(r)}, \Lambda\right) .
$$

On the other hand we get a homomorphism $f^{+}: A^{+} \rightarrow B^{+}$of supplemented algebras, and so a homomorphism

$$
\operatorname{TOR}_{n}^{A^{+}}\left(B^{(r)}, \Lambda\right) \rightarrow \operatorname{TOR}_{n}^{B^{+}}\left(B^{(r)}, \Lambda\right) .
$$

By composition we now have the required homomorphism

$$
T(f): T(A) \rightarrow T(B) .
$$

7.1. THEOREM. There are functor isomorphisms

$$
\begin{aligned}
& \operatorname{TOR}_{0}^{A^{+}}\left(A^{(r)}, \Lambda\right) \cong \operatorname{Coker}\left[D_{0} V^{r+1}(A) \rightarrow D_{0} V^{r}(A)\right], \\
& \operatorname{TOR}_{1}^{A^{+}}\left(A^{(r)}, \Lambda\right) \cong \operatorname{Ker}\left[D_{0} V^{r+1}(A) \rightarrow D_{0} V^{r}(A)\right] .
\end{aligned}
$$


REMARK. In view of (6.6) this theorem provides explicit formulae for certain homology groups.

Before giving the proof of 7.1 we note that for $r=1$ we have on the one hand

$$
\operatorname{TOR}_{n}^{A^{+}}(A, \Lambda)=\operatorname{TOR}_{n+1}^{A^{+}}(\Lambda, \Lambda),
$$

while on the other hand

$$
\begin{aligned}
\operatorname{Ker}\left[D_{0} V^{2}(A)\right. & \left.\rightarrow D_{0} V^{1}(A)\right]=D_{1} U^{2}(A), \\
\operatorname{Coker}\left[D_{0} V^{2}(A) \rightarrow D_{0} V^{1}(A)\right] & =U^{2}(A) .
\end{aligned}
$$

Hence we get

7.2. COROLlary.

(i) $\operatorname{TOR}_{1}^{A^{+}}(\Lambda, \Lambda)=A / A^{2}$,

(ii) $\operatorname{TOR}_{2}^{A^{+}}(\Lambda, \Lambda)=A_{0}^{2} \cap A_{1} / A_{0} A_{1}+A_{1} A_{0}$,

whenever $A \cong A_{0} / A_{1}$, with $A_{0}$ a projective algebra.

7.2 (i) is well known. It is the analogue of the equation

$$
H_{1}(G, Z)=G /(G, G)
$$

for the homology group of a group $G$ in dimension 1 .

7.2 (ii) is the analogue of the Hopf equation

$$
H_{2}(G, Z)=(F, F) \cap K /(K, F),
$$

which holds whenever $G$ is represented as the quotient group of a free group $F$ with kernel $K$. (See Introduction, equation (3).)

Proof of 7.1. Let $\mathbf{C}$ be the complex with $C_{0}=A^{+}, C_{1}=A, C_{n}=0$ otherwise, and $C_{1} \rightarrow C_{0}$ the inclusion map $g_{0, A^{+}}^{1}$ Then for $i=0,1$ the $i$ th homology module of the complex $A^{(r)} \otimes_{A^{+}} \mathfrak{C}$ is $\operatorname{TOR}_{i}^{A^{+}}\left(A^{(r)}, \Lambda\right)$. But by (5.3) $g_{r, A}^{r+1}=i_{A}^{r} \otimes_{A^{+}} g_{0, A^{*}}^{1}$ Hence

$$
\begin{aligned}
\operatorname{Ker} g_{r, A}^{r+1} & =\operatorname{TOR}_{1}^{A^{+}}\left(A^{(r)}, \Lambda\right), \\
\operatorname{Coker} g_{r, A}^{r+1} & =\operatorname{TOR}_{0}^{A^{+}}\left(A^{(r)}, \Lambda\right) .
\end{aligned}
$$

Let now $H_{0}(\mathbf{A}) \cong A$ be a projective presentation of $A$. We have a commutative diagram

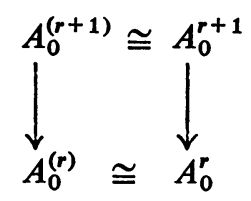

whose rows induce the isomorphisms 


$$
A^{(r+1)} \cong D_{0} V^{r+1}(A), \quad A^{(r)} \cong D_{0} V^{r}(A)
$$

of 6.1, and whose columns induce the homomorphisms

$$
A^{(r+1)} \rightarrow A^{(r)}, \quad D_{0} V^{r+1}(A) \rightarrow D_{0} V^{r}(A) .
$$

We thus obtain natural isomorphisms

$$
\begin{aligned}
\operatorname{Ker} g_{r, A}^{r+1} & \cong \operatorname{Ker}\left[D_{0} V^{r+1}(A) \rightarrow D_{0} V^{r}(A)\right], \\
\operatorname{Coker} g_{r, A}^{r+1} & \cong \operatorname{Coker}\left[D_{0} V^{r+1}(A) \rightarrow D_{0} V^{r}(A)\right] .
\end{aligned}
$$

Comparison with (7.1) now gives the theorem.

From 6.1 we obtain, by tensoring the monomorphism $D_{1} U^{r}(A) \rightarrow A^{(r)}$ with $A$, a homomorphism

$$
D_{1} U^{r}(A) \otimes_{A^{+}} A \rightarrow A^{(r+1)} .
$$

Similarly the epimorphism $A^{(r)} \rightarrow A^{r}$ gives rise to an epimorphism

$$
\sigma_{A^{\prime}}: A^{(r)} \otimes_{A^{+}} A \rightarrow A^{r} \otimes_{A^{+}} A,
$$

and

$$
\operatorname{Ker} \sigma_{A}=\operatorname{Im}\left[D_{1} U^{r}(A) \otimes_{A}+A \rightarrow A^{(r+1)}\right] .
$$

As $\operatorname{Ker} \sigma_{A} \subseteq \operatorname{Ker} g_{1, A}^{r+1} \cong D_{1} U^{r+1}(A)$ we now obtain a homomorphism

$$
D_{1} U^{r}(A) \otimes_{A^{+}} A \rightarrow D_{1} U^{r+1}(A) \text {. }
$$

All these homomorphisms are natural. On the other hand we may consider $\operatorname{TOR}_{n}^{A^{+}}\left(A^{r}, \Lambda\right)$ as a functor of $\mathfrak{C}$ in the category of $\Lambda$-modules. We then have

7.3. THEOREM. There are functor isomorphisms

(i) $\operatorname{Coker}\left[\operatorname{TOR}_{2}^{\Lambda^{+}}\left(A^{(r)}, \Lambda\right) \rightarrow \operatorname{TOR}_{2}^{\Lambda^{+}}\left(A^{r}, \Lambda\right)\right] \cong \operatorname{Ker}\left[D_{1} U^{r}(A) \otimes_{\Lambda^{+}} A \rightarrow D_{1} U^{r+1}(A)\right]$,

(ii) $\operatorname{TOR}_{1}^{\Lambda^{+}}\left(A^{r}, \Lambda\right) \cong \operatorname{Coker}\left[D_{1} U^{r}(A) \otimes_{A^{+}} A \rightarrow D_{1} U^{r+1}(A)\right]$,

(iii) $\operatorname{TOR}_{0}^{A^{+}}\left(A^{r}, \Lambda\right) \cong \operatorname{Ker}\left[U^{r+1}(A) \rightarrow U^{r}(A)\right]$.

Proof. From the exact sequence

$$
0 \rightarrow D_{1} U^{r}(A) \rightarrow A^{(r)} \rightarrow A^{r} \rightarrow 0
$$

we obtain the exact homology sequence

$$
\cdots \rightarrow \operatorname{TOR}_{1}^{\Lambda^{+}}\left(A^{(r)}, A\right) \rightarrow \operatorname{TOR}_{1}^{\Lambda^{+}}\left(A^{r}, A\right) \rightarrow D_{1} U^{r}(A) \otimes_{1^{+}} A \rightarrow A^{(r)} \otimes_{1^{+}} A \rightarrow \cdots
$$

and so, as $\operatorname{TOR}_{1}^{A^{+}}(X, A)=\operatorname{TOR}_{2}^{\Lambda^{+}}(X, \Lambda)$, an isomorphism

$$
\operatorname{Coker}\left[\operatorname{TOR}_{2}^{A^{+}}\left(A^{(r)}, \Lambda\right) \rightarrow \operatorname{TOR}_{2}^{\Lambda^{+}}\left(A^{r}, \Lambda\right)\right] \cong \operatorname{Ker}\left[D_{1} U^{r} A \otimes_{A^{+}} A \rightarrow A^{(r+1)}\right] \text {. }
$$

But the right hand side here coincides with the right hand side in (i). 
For (ii) and (iii) we consider the commutative diagram with exact rows and columns

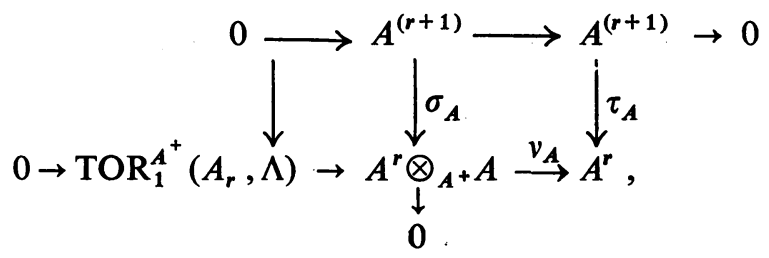

where $v_{A}=\iota_{A^{r}} \otimes_{A^{+}} g_{0, A}^{1}$ and so

$$
\operatorname{TOR}_{0}^{A^{+}}\left(A^{r}, \Lambda\right) \cong \operatorname{Coker} v_{A}=A^{r} / A^{r+1},
$$

which is the isomorphism (iii). From (7.3) we get an exact sequence

$$
0 \rightarrow \operatorname{Ker} \sigma_{A} \rightarrow \operatorname{Ker} \tau_{A} \rightarrow \operatorname{TOR}_{1}^{A^{+}}\left(A^{r}, \Lambda\right) \rightarrow 0 .
$$

As $\operatorname{Ker} \tau_{A} \cong D_{1} U^{r+1}(A)$, and by (7.2), we now get (ii).

$\S 7.2$ is also a corollary of 7.3. We only have to take again the case $r=1$.

\section{Examples.}

8.1. Proposition. If the algebra $A$ has an identity $e$ then for all $r$,

$$
\begin{aligned}
& D_{1} U^{r}(A)=0, \\
& D_{0} V^{r}(A)=A^{r}=A .
\end{aligned}
$$

Proof. The map

$$
a \rightarrow e \otimes \cdots \otimes e \otimes a
$$

defines a homomorphism $f: A \rightarrow A^{(r)}$ of $\Lambda$-modules and $f g_{1, A}^{r}$ is the identity map of $A^{(r)}$. Hence $\operatorname{Ker} g_{1, A}^{r}=0$. Now apply 6.1, and observe that $A^{r}=A$.

If $J, K$ are ideals of a commutative algebra $A$ we write $[J: K]$ for the ideal consisting of the elements $a \in A$ with $a K \subseteq J$.

8.2. Proposition. If $A$ is an algebra on a single generator, then for all $r \geqq 1$

$$
\begin{aligned}
& D_{0} V^{r+1}(A) \cong A, \\
& D_{1} U^{r+1}(A) \cong\left[0: A^{r}\right]
\end{aligned}
$$

(isomorphisms of two sided $A^{+}$-modules).

Proof. Let $A_{0}$ be the free algebra on a single generator $x$, and let $\theta: A_{0} \rightarrow A$ be a homomorphism of algebras with $\theta(x)$ as generator of $A$. Then $\theta$ is an epimorphism. Write $\operatorname{Ker} \theta=A_{1}$.

The map $y \rightarrow y x^{r}\left(y \in A_{0}\right)$ gives rise to an isomorphism $f: A_{0} \cong A_{0}^{r+1}$ of two sided $A_{0}^{+}$-modules, and $f$ induces isomorphisms 


$$
\begin{aligned}
A_{1} & \cong A_{0}^{r} A_{1}=V_{1}^{r+1}(\mathrm{~A}), \\
{\left[A_{1}: A_{0}^{r}\right] } & \cong A_{0}^{r+1} \cap A_{1} .
\end{aligned}
$$

We thus get isomorphisms

$$
\begin{gathered}
A_{0} / A_{1} \cong A_{0}^{r+1} / V_{1}^{r+1}(\mathbf{A})=D_{0} V^{r+1}(A), \\
{\left[A_{1}: A_{0}^{r}\right] / A_{1} \cong A_{0}^{r+1} \cap A_{1} / V_{1}^{r+1}(\mathbf{A})=D_{1} U^{r+1}(A) .}
\end{gathered}
$$

On the other hand $\theta$ gives rise to isomorphisms

$$
\begin{gathered}
A_{0} / A_{1} \cong A, \\
{\left[A_{1}: A_{0}^{r}\right] / A_{1} \cong\left[0: A^{r}\right] .}
\end{gathered}
$$

The isomorphisms of 8.2 are the compositions of the inverses in (8.1) with those in (8.2). They are isomorphisms of $A_{0}^{+}$-modules, and so, as everything is annihilated by $A_{1}$, of $A^{+}$-modules.

The last example is to illustrate the fact that $D_{1} U^{r}, D_{0} V^{r}$ are not additive. Let, for $r \geqq 2, \mathcal{F}$ be the set of maps of the set $\{1,2, \cdots, r\}$ onto the set $\{1,2\}$. Let $R$ be a ring, $M_{1}, M_{2}$ two sided $R$-modules, and let $f \in \mathfrak{F}$. We then write

$$
L^{R}(f)=M_{f(1)} \otimes_{R} M_{f(2)} \otimes \cdots \otimes_{R} M_{f(r)}
$$

and we denote the direct sum of the modules $L^{R}(f)$, with $f$ running over $\mathfrak{F}$, by $L_{r}^{R}\left(M_{1}, M_{2}\right)$.

8.3. Proposition. Let $A=A_{1}+A_{2}$ (direct sum of ideals). Then for $r \geqq 2$,

(i) $D_{0} V^{r}(A) \cong D_{0} V^{r}\left(A_{1}\right)+D_{0} V^{r}\left(A_{2}\right)+L_{r}^{\Lambda}\left(A_{1} / A_{1}^{2}, A_{2} / A_{2}^{2}\right)$,

(ii) $D_{1} U^{r}(A) \cong D_{1} U^{r}\left(A_{1}\right)+D_{1} U^{r}\left(A_{2}\right)+L_{r}^{\Lambda}\left(A_{1} / A_{1}^{2}, A_{2} / A_{2}^{2}\right)$,

(isomorphisms of algebras and of two sided $A^{+}$-modules).

Proof. We use 6.1. $A_{1}$ and $A_{2}$ are two sided $A^{+}$-modules, and so

$$
A^{(r)} \cong A_{1}^{(r)}+A_{2}^{(r)}+L_{r}^{A^{+}}\left(A_{1}, A_{2}\right) \quad \text { (direct sum of ideals). }
$$

As $A_{1} A_{2}=A_{2} A_{1}=0$ the last term on the right has zero multiplication and lies in $\operatorname{Ker} g_{1, A}^{r}$.

8.3 now follows once we have shown that

$$
L_{r}^{A^{+}}\left(A_{1}, A_{2}\right) \cong L_{r}^{\Lambda}\left(A_{1} / A_{1}^{2}, A_{2} / A_{2}^{2}\right) .
$$

One verifies easily that

$$
\operatorname{Ker}\left[A_{1} \otimes_{\Lambda} A_{2} \rightarrow A_{1} / A_{1}^{2} \otimes_{\Lambda} A_{2} / A_{2}^{2}\right]=\operatorname{Ker}\left[A_{1} \otimes_{\Lambda} A_{2} \rightarrow A_{1} \otimes_{A^{+}} A_{2}\right]
$$

whence

$$
A_{1} \otimes_{A^{+}} A_{2} \cong A_{1} / A_{1}^{2} \otimes_{\mathrm{\Lambda}} A_{2} / A_{2}^{2}
$$

and similarly on interchange of subscripts. But this implies (8.3). 


\section{REFERENCES}

1. R. Baer, Representations of groups as quotient groups. I-III, Trans. Amer. Math. Soc. 58 (1945), 295-419.

2. H. Cartan and S. Eilenberg, Homological algebra, Princeton Univ. Press, Princeton, N.J., 1956.

3. P. J. Higgins, Groups with multiple operators, Proc. London Math. Soc. (3) 6 (1956), 366-416.

4. H. Hopf, Fundamentalgruppe und zweite Bettische Gruppe, Comment. Math. Helv. 14 (1942), 257-309.

5. A Fröhlich, On groups over a d.g. near-ring. II. Categories and functors, Quart. J. Math. Oxford Ser. (2) 2 (1960), 211-228.

6. - Non-Abelian homological algebra. I, Proc. London Math. Soc. (3) 11 (1961), 239-275; II, Proc. London Math. Soc. (3) 12 (1962), 1-28.

King's College,

LONDON, ENGLAND

California Institute of Technology,

Pasadena, California 